\title{
Expression of Beclin-1 in the
} Microenvironment of Invasive Ductal Carcinoma of the Breast: Correlation with Prognosis and the Cancer-Stromal Interaction

\author{
Akemi Morikawa ${ }^{1,2}$, Tamotsu Takeuchi $^{3 *}$, Yusuke Kito $^{3}$, Chiemi Saigo $^{3}$, \\ Takuji Sakuratani $^{1}$, Manabu Futamura ${ }^{1,4}$, Kazuhiro Yoshida ${ }^{1}$ \\ 1 Department of Surgical Oncology, Gifu University Graduate School of Medicine, Gifu, Japan, \\ 2 Department of Frontier Science for Surgical Oncology, Gifu University Graduate School of Medicine, Gifu, \\ Japan, 3 Department of Pathology and Translational Research, Gifu University Graduate School of \\ Medicine, Gifu, Japan, 4 Department of Breast and Molecular Oncology, Gifu University Graduate School of \\ Medicine, Gifu, Japan \\ * takeutit08@ gmail.com
}

Citation: Morikawa A, Takeuchi T, Kito Y, Saigo C, Sakuratani T, Futamura M, et al. (2015) Expression of Beclin-1 in the Microenvironment of Invasive Ductal Carcinoma of the Breast: Correlation with Prognosis and the Cancer-Stromal Interaction. PLOS ONE 10 (5): e0125762. doi:10.1371/journal.pone.0125762

Academic Editor: Rajeev Samant, University of Alabama at Birmingham, UNITED STATES

Received: November 13, 2014

Accepted: March 26, 2015

Published: May 8, 2015

Copyright: @ 2015 Morikawa et al. This is an open access article distributed under the terms of the Creative Commons Attribution License, which permits unrestricted use, distribution, and reproduction in any medium, provided the original author and source are credited.

Data Availability Statement: All relevant data are within the paper and its Supporting Information files.

Funding: This work was supported by Ministry of Education of Japan (KAKEN 24590384 and 25860267), and Yakult Honsha Co., Ltd., Tokyo. The funders had no role in study design, data collection and analysis, decision to publish, or preparation of the manuscript.

Competing Interests: One of the authors (Akemi Morikawa) was a member of the Endowed Chair of Gifu University, to which unrestricted research

\section{Abstract}

We examined the pathobiological properties of beclin-1, which is a key regulator of autophagosome formation in invasive ductal carcinoma of the breast, with a particular focus on the cancer microenvironment. Immunohistochemistry demonstrated that cancer cells and stromal mesenchymal cells expressed beclin-1 in 68 and 38 of 115 invasive ductal cancers, respectively. Expression of beclin-1 in cancer or stromal cells alone did not correlate with patient prognosis. In contrast, loss of beclin-1 in cancer cells and overexpression in stromal mesenchymal cells was associated with local cancer recurrence, postoperative lymph node metastasis, and a poor disease-free survival rate. A comprehensive gene expression analysis was performed on a co-culture of breast cancer cells and mesenchymal stromal cells, that latter of which either expressed beclin-1 or was depleted of beclin-1 by siRNA. Notably, siRNA-mediated downregulation of beclin-1 in mesenchymal cells co-cultured with breast cancer cells decreased the levels of various pro-inflammatory cytokines, their receptors, and collagen receptors. Quantitative reverse transcription polymerase chain reaction analysis confirmed that reduction of stromal beclin-1 expression decreased the expression of $I L$ $1 \beta$ and collagen receptor discoidin domain receptor 2 (DDR2). Microenvironmental IL- $1 \beta$ is believed to play an important role in tumor invasion. Recent work has also indicated that overexpression of DDR2 contributes to breast cancer invasion and lymph node metastasis. Taken together, these findings indicate beclin-1 expression in the stroma might be important for shaping the breast cancer microenvironment and thus could be a potent molecular target in patients with invasive ductal carcinoma of the breast. 
funding will be donated by the commercial company Yakult Honsya Co., Ltd., Tokyo. This does not alter the authors' adherence to PLOS ONE policies on sharing data and materials.

\section{Introduction}

Autophagy is the process of self-digestion in which lysosomal degradation is used to maintain cellular viability during periods of metabolic stress such as starvation [1,2]. During carcinogenesis, autophagy is important for tumor cell survival under starvation conditions. Paradoxically, autophagy defects are also associated with increasing carcinogenesis [3,4]. Beclin-1 is important at major step in autophagic pathways, from autophagosome formation, to autophagosome/endosome maturation. Beclin-1 is important at the major step in autophagic pathways, from autophagosome formation to autophagosome/endosome maturation [5,6]. Thus, beclin1 is a key protein and reliable biomarker of autophagy in various cancers. Notably, although beclin-1 is normally expressed in the ductal epithelial cells of mammary glands, many breast cancer cells lack beclin-1 expression due to a combination of monoallelic deletion and epigenetic silencing of the beclin-1 gene [5, 7]. Exogenous expression of beclin-1 in MCF-7 breast cancer cells promoted autophagy, inhibited cell growth, and decreased tumorigenesis in nude mice [5]. However, beclin-1-dependent autophagy is required for the tumorigenicity of breast cancer stem-like/progenitor cells [8]. Therefore, beclin-1 expression may have a dual role in breast carcinogenesis, acting both to promote and to suppress tumor progression.

Expression of beclin-1 in the tumor stroma also has an important role in the development of various cancers. The stromal cells play a crucial role in the progression of invasive breast cancer [9]. Autophagy in the cancer-associated stroma supports cancer progression metabolically through increasing glycolysis and ketone production in the tumor microenvironment [10]. Moreover, stromal autophagy plays a critical role in maintaining the tumor microenvironment to facilitate the growth of neighboring cancer cells [11]. Despite these findings, which highlight the important role of beclin-1 expression in the cancer-stromal niche, the combined effects of beclin-1 expression in tumor and stromal cells on prognosis of breast cancer patients remain unclear.

In this study, we first asked whether beclin-1 expression in invasive ductal carcinoma cells and/or stromal cells correlated with the prognosis of breast cancer patients. We subsequently examined the molecular background, which might reflect the clinicopathological findings, using a co-culture system comprising breast cancer- and bone marrow-derived mesenchymal cells.

\section{Materials and Methods}

\section{Ethical statements}

The paraffin-embedded tissues surgically resected from the patients were used as a retrospective study after its use for diagnosis. The need for written informed consent was waived by the Institutional Review Board of the Gifu University Graduate School of Medicine. Instead, the Institutional Review Board requested us to inform the patients that they could refuse to use their tissue specimens for this study, if they did not want to participate in the present study. The present study was conducted in accordance with the ethical standards of the Helsinki Declaration in 1975, after approval of the Institutional Review Board of the Gifu University Graduate School of Medicine (a specific approval number 25-81).

\section{Antibodies and immunohistochemical staining}

A mouse-specific monoclonal antibody (clone $4 \mathrm{H} 10$ ) and a conventional rabbit antibody to beclin-1 were purchased from Novus Biologicals (Littleton, CO, USA) and Genetex (San Antonio, TX, USA), respectively. A normal rabbit antibody was also prepared in our laboratory. 
Archived pathological tissue specimens from 115 invasive ductal carcinomas were used in this study. All tissue specimens were obtained surgically, fixed in $10 \%$ buffered formalin, and embedded in paraffin. Tissues were immunostained with antibodies using the ImmPRESS polymerized reporter enzyme staining system (Vector laboratories, Inc., Burlingame, CA, USA) as previously reported [12]. The tissue specimens were considered positive if the cancer cells or mesenchymal stromal cells exhibited more than $5 \%$ staining.

\section{Statistical analysis}

Curves for disease free survival were drawn using the Kaplan-Meier method and the differences in survival rates were compared using the log-rank test for univariate survival analysis. A p value of $<0.05$ was considered statistically significant.

\section{Cell culture and siRNA-mediated RNA interference}

UE6E7T-2 human bone marrow-derived mesenchymal cells, which have been used as a model of cancer-associated stromal mesenchymal cells $[13,14]$, were obtained from the RIKEN Biosource Center (Tsukuba, Japan). The MCF-7 and MDA-MB-157 breast cancer cell lines [15, 16], which are known to express little or no beclin-1 [5], were obtained from the Japan Health Science Research Resources Bank (Osaka, Japan) and American Type Culture Collection (Rockville, MD, USA), respectively. UE6E7T-2, MCF-7, and MDA-MB-157 were passaged in our laboratory for no more than 6 months after resuscitation.

The detailed procedure for siRNA silencing of a target gene has been described previously [12]. In this study, we employed the beclin-1 siRNA: 5'-CAGUUACAGAUGGAGCUAAtt-3', which is located downstream from the initiation codon of the human beclin-1 coding region and has been used in previous reports.

We also used two other siRNA sequences, $5^{\prime}$-CUCAGGAGAGGAGCCAUUUtt- $3^{\prime}$ and $5^{\prime}$ GAUUGAAGACACAGGAGGCUU- $3^{\prime}$, for silencing the beclin-1 gene and then examined the expressions of $I L-1 \beta, D D R 2$, and $I L-10 R B$. A green fluorescent protein (GFP) siRNA duplex with the target sequence 5'-CGGCAAGCUGACCCUGAAGUUCAU-3' was used as a non-silencing control. siRNAs were transfected into UE6E7T-2 cells using lipofectamine RNAiMAX in accordance with the manufacturer's instructions (Invitrogen, Carlsbad, CA, USA). At $48 \mathrm{~h}$ after transfection, the cells were used for subsequent studies.

Beclin-1 siRNA-treated or control UE6E7T-2 cells $\left(1 \times 10^{4}\right)$ and MCF-7 or MDA-MB-157 cells $\left(1 \times 10^{4}\right)$ were co-cultured in 24 well plates. After $48 \mathrm{~h}$, total RNA was extracted from the co-cultured cells using RNeasy spin columns kit (Qiagen, Santa Clarita, CA, USA).

\section{Western blotting}

Western blotting was performed according to the previously described method [17], in accordance with the proposal by Towbin et al. [18]. Cells were disrupted in Radio-Immunoprecipitation Assay (RIPA) buffer (Sigma-Aldrich, St. Louis, MO, USA), composed of $150 \mathrm{mM} \mathrm{NaCl}$, $1.0 \%$ IGEPAL CA-630, $0.5 \%$ sodium deoxycholate, $0.1 \%$ SDS, and $50 \mathrm{mM}$ Tris (pH 8.0) supplemented with a protease inhibitor cocktail (Nacalai Tesque, Kyoto, Japan), on ice for $15 \mathrm{~min}$ and then incubated at room temperature for $30 \mathrm{~min}$ with $0.25 \mathrm{U} / \mu \mathrm{L}$ of benzonase (Sigma-Aldrich). Then, these total cell lysates were mixed 1:1 with $2 \times$ SDS sample buffer (4\% SDS, $20 \%$ glycerol, $0.12 \mathrm{M}$ Tris (pH 6.8), $0.2 \mathrm{M} \mathrm{DTT}$, and $0.02 \%$ bromophenol blue), incubated for $3 \mathrm{~min}$ at $95^{\circ} \mathrm{C}$, and subjected to SDS-PAGE. The separated proteins were transferred onto polyvinylidene difluoride membranes (Millipore Co., Bedford, MA, USA) and probed with an anti-beclin-1 antibody (clone $4 \mathrm{H} 10$ ), anti-IL-1 $\beta$ antibody (Proteintech, Chicago, IL, USA), anti-actin antibody 
(Sigma-Aldrich), or anti-GAPDH antibody (Sigma-Aldrich). Immunoreactivity was assessed using a western blotting detection kit (Promega, Madison, WI, USA).

\section{cDNA microarray assay, reverse transcription polymerase chain reaction, and quantitative real-time reverse transcription polymerase chain reaction}

We utilized the Human Whole Genome DNA Microarray system (SurePrint G3 Human $8 \times 60 \mathrm{~K}$ ver. 2.0, Agilent Technologies, Santa Clara, CA) to obtain the altered gene expression profile for co-cultured MCF-7 and UE6E7T-2 cells in which the beclin-1 gene was silenced by siRNA. cDNA synthesis from the total RNA and subsequent PCR were performed using a reverse transcription polymerase chain reaction (RT-PCR) kit (Takara, Ohtsu, Japan). The procedure was performed according to the manufacturer's instructions, as previously described [17].

The microarray data has been deposited to the GEO database with accession number GSE66154.

The Agilent-039494 SurePrint G3 Human GE v2 8x60K Microarray 039381 platform was used (Agilent, Santa Clara, CA, USA). Scanning and image analysis were performed using a DNA Microarray Scanner (Agilent). Raw fluorescence intensities were quantified and normalized using Agilent Feature Extraction Software (Agilent Feature Extraction 10.7.3.1). Normalization was performed with GeneSpring GX (Agilent). For processing, data were normalized by the percentile shift (all samples were normalized to the signal value corresponding to the 75th percentile of all of probes on the microarray).

To obtain the hierarchical combined tree for the 22 extracted genes reported to be related to inflammation or stromal reconstitution, we employed the GeneSpring Multi-Omic Analysis System (Agilent).

Real-time PCR reactions were performed using the SYBR Green reaction kit according to the manufacturer's instructions (Roche Diagnostics, GmbH, Mannheim, Germany) in a LightCycler (Roche Diagnostics). cDNA ( $2 \mu \mathrm{L}$ each) was diluted to a volume of $20 \mu \mathrm{L}$ with the PCR mix containing a final primer concentration of 0.2 pmol.

The following primers were used for real-time RT-PCR: qPCR primers for human beclin1-forward 5'-ACCGTGTCACCATCCAGGAA-3', beclin-1-reverse 5'-GAAGCTGTTGGC ACTTTCTGT-3'; GAPDH-forward 5'-GAAATCCCATCACCATCTTCCAGG-3', GAPDHreverse $5^{\prime}$-GAGCCCCAGCCTTCTCCATG- ${ }^{\prime}$; IL-1 $1 \beta$-forward $5^{\prime}$-AAAGAGGCACTGGCAG AA- $3^{\prime}$, IL-1 1 -reverse $5^{\prime}$-AGCTCTGGCTTGTTCCTCAC-3'; DDR2-forward $5^{\prime}$-AGTCAG TGGTCAGAGTCCACAGC-3', DDR2-reverse 5'-CAGGGCACCAGGCTCCATC- ${ }^{\prime}$; and IL10RB (IL-10 receptor, subunit beta)-forward 5'-GGGGTCGTGTGCTTGGAG-3', IL10RBreverse $5^{\prime}$-GGTACCATTCCCAATGCTGA- $3^{\prime}$.

To ensure that the SYBR green was not incorporated into primer dimers or non-specific amplicons during the real-time PCR runs, the PCR products were analyzed by polyacrylamide gel electrophoresis in preliminary experiments. Single bands of the expected sizes were obtained in all instances. The samples were cultured in triplicate, and the expression of each target gene was analyzed by the $2^{-\Delta \Delta C T}$ method of Livak and Schmittgen [19] using the LightCycler system. The $\Delta \mathrm{C}_{\mathrm{T}}$ values were normalized to GAPDH for each triplicate set in both the si-GFPtreated (control) and si-beclin-1-treated groups. The values for the si-beclin-1-treated group were then calculated for each target gene as the fold change relative to the mean values for the si-GFP-treated group (control; set to 1.0). Then, the standard deviations were computed for the triplicate sets, namely, the 3 target genes and the fold changes are presented. In addition, 
Student's t-tests were performed to determine significant differences. $\mathrm{P}<0.05$ was considered to be statistically significant.

\section{Results}

\section{Expression of beclin-1 in invasive carcinoma tissue specimens}

In this study, we predominantly used a murine monoclonal antibody to beclin- 1 (clone $4 \mathrm{H} 10$ from Novus Biologicals) for immunohistochemical staining, but we also used another commercially available conventional rabbit antibody to beclin-1 on several tissue specimens to confirm the results. We obtained similar results with both antibodies. Significant immunoreactivity was not found using the control rabbit antibody.

Representative results from the immunohistochemical staining are shown in Fig 1. In this study, we examined beclin-1 expression in archived pathological tissue specimens from patients with invasive ductal carcinoma. Immunoreactivity of the anti-beclin- 1 antibody was observed in the cancer cells of 68 of 115 tissue specimens and in the mesenchymal stromal cells of 38 of 115 invasive carcinoma tissue specimens (Fig 2). The absence of beclin-1 expression in the cancer cells alone was not significantly associated with a poor patient prognosis. Stromal beclin-1 expression was most often associated with a poor patient prognosis but this was not statistically significant. In contrast, lack of beclin-1 expression in cancer cells with concomitant stromal beclin-1 expression was linked to local cancer recurrence and postoperative lymph node metastasis, and thus was significantly associated with a poor disease-free survival rate (Fig 2).

\section{siRNA-mediated silencing of beclin-1 gene expression in mesenchymal stromal cells downregulated the expression of the $I L-1$ agonist and $D D R 2$ genes, but upregulated the IL-10RB gene in a MCF-7 or MDA-MB- 157 and mesenchymal stromal cell co-culture system}

Next, we examined the molecular pathway responsible for the aggressive phenotype of beclin1-negative cancer cells that have concomitant stromal beclin-1 expression. Our preliminary experiments demonstrated that MCF-7 and MDA-MB-157 breast cancer cells express low levels of beclin-1, as previously reported [5]

In contrast, several mesenchymal cell lines abundantly express beclin-1. In this study, we employed a bone marrow-derived, mesenchymal UE6E7T-2 cell line as a model of stromal cells for the following reasons. First, recent studies revealed that bone marrow-derived mesenchymal cells are important for the cancer-stromal microenvironment. Second, the usefulness of UE6E7T-2 mesenchymal cells and its series cells as a model of cancer stromal mesenchymal cells is well established $[13,14]$. Third, preliminary experiments demonstrated that UE6E7T-2 cells allow regulation of beclin-1 expression by siRNA-mediated silencing, which was performed in this study.

Genes that showed a greater than 2-fold difference in expression (by cDNA microarray assay, S1 and S2 Datasets) between the co-cultures of MCF-7 cells with either beclin-1-expressing or beclin-1-downregulated UE6E7T-2 cells are summarized in Fig 3.

Notably, downregulation of beclin-1 in UE6E7T-2 cells decreased the expression of several pro-inflammatory cytokines and agonists such as IL-1 , interleukin 18 receptor 1 (IL18R1), and $g p 130$, but increased the expression of anti-inflammatory genes such as interleukin-10 receptor beta (IL-10RB) and interleukin 18 binding protein (IL18BP). Moreover, downregulation of beclin-1 decreased $D D R 2$ expression, which was recently shown to be critical for stabilizing SNAIL1 to promote breast cancer invasion and lymph node metastasis [20, 21]. In additional 


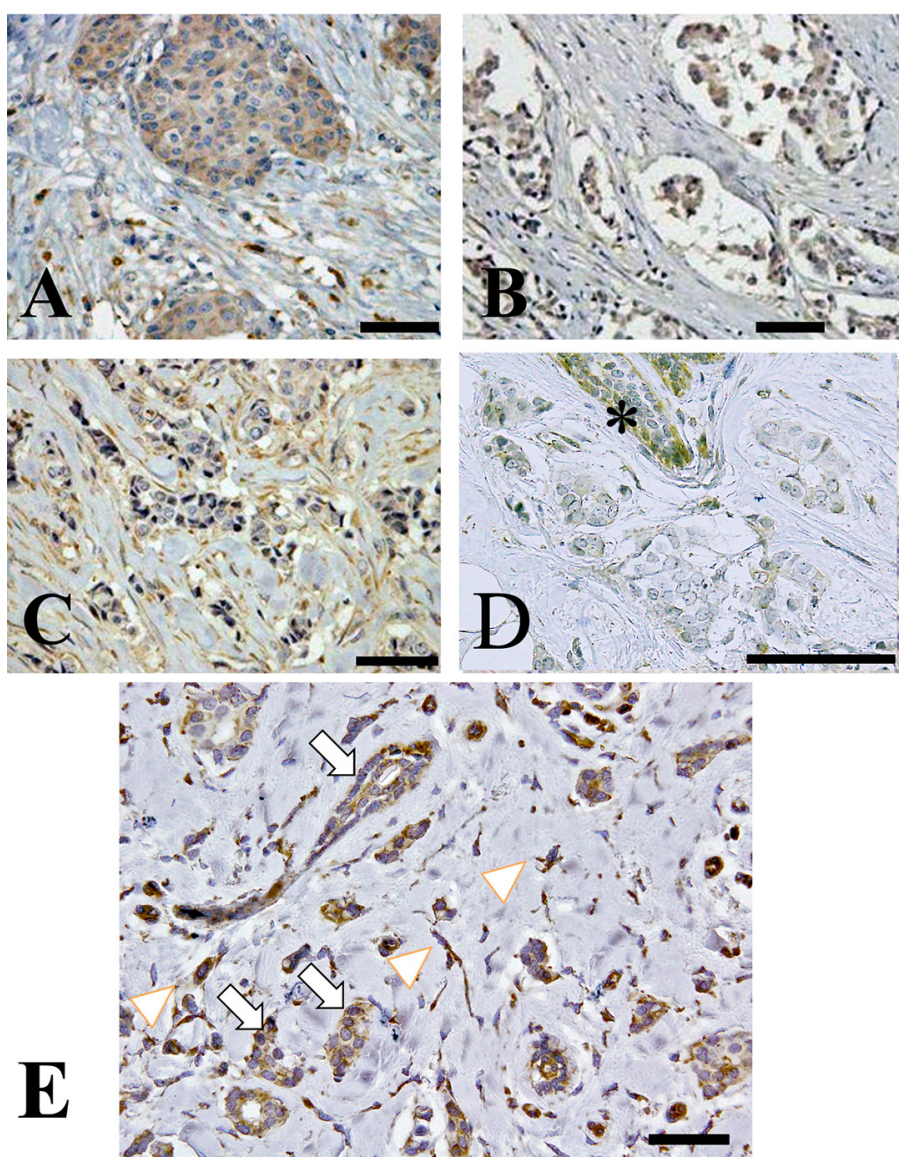

Fig 1. Representative immunohistochemical staining. (A) Both invasive ductal carcinoma and cancer stromal cells showing immunoreactivity with a specific monoclonal antibody to beclin-1. (B) Invasive ductal carcinoma cells, not the stromal cells, exhibiting beclin-1 immunoreactivity. (C) Significant immunoreactivity is not observed in the invasive ductal cancer cells, whereas the cancer-associated stromal cells exhibit beclin-1 immunoreactivity. (D) Neither invasive ductal cancer cells nor stromal cells showing beclin-1 immunoreactivity. Note the beclin-1 immunoreactivity in non-tumorous ductal epithelial cells (indicated by asterisk). (E) Cancer and stromal cells exhibiting beclin-1 immunoreactivity are indicated by arrows and arrow heads, respectively. Scales bars: $100 \mu \mathrm{m}$.

doi:10.1371/journal.pone.0125762.g001

experiments, the viability of co-cultured cells exceeded $95 \%$ in both si-GFP- and si-beclin1-treated groups. The ratios of the MCF-7 and UE6E7T-2 populations were preserved in siGFP- and si-beclin-1-treated groups (S4 Fig).

In summary, we confirmed the microarray data that were focused on the $I L-1 \beta, I L-10 R B$, and $D D R 2$ genes. RT-PCR analysis demonstrated that the downregulation of beclin-1 in UE6E7T- 2 cells significantly abrogated the expressions of $I L-1 \beta$ and $D D R 2$, but increased the expression of $I L-10 R B$ in co-cultured MCF-7 and UE6E7T-2 cells (Fig 4). We performed three independent, quantitative RT-PCR experiments using different MCF-7-UE6E7T-2 co-culture systems and obtained similar results. We also found that $I L-1 \beta$ and $D D R 2$ gene expressions were decreased, whereas $I L-10 R B$ gene expression was increased, by the downregulation of UE6E7T-2-beclin-1 in a co-culture system using MDA-MB-157 cells, similar to that found using MCF-7 cells (S1 Fig). We also employed two other siRNA sequences to exclude off-target effects and obtained similar results (representative data are shown in S2 and S3 Figs). 


\section{Beclin-1 immunoreactivity Stromal cells}

\author{
positive
}

\section{Cancer cell \\ positive}

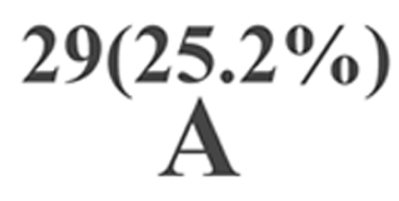

negative

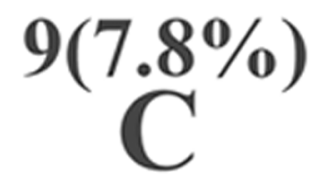

negative

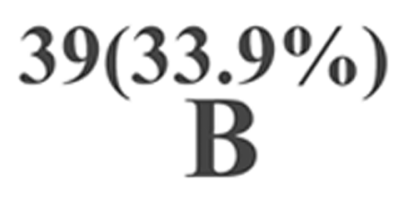

$\stackrel{38(33.0 \%)}{\mathrm{D}}$

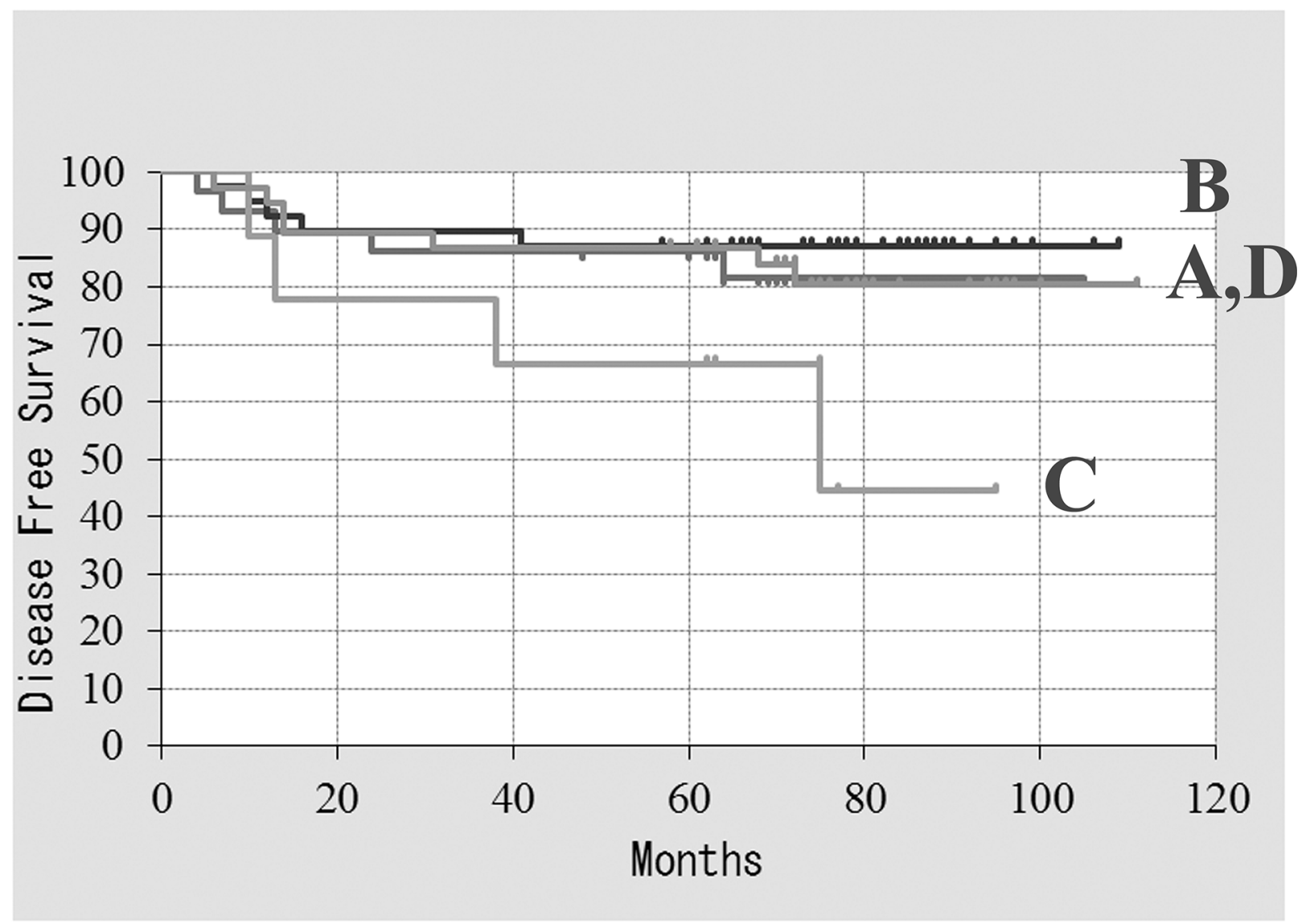

Fig 2. Disease-free survival curves according to beclin-1 expression in cancer and stromal cells. Lack of beclin-1 expression in cancer cells with concomitant stromal beclin-1 expression (indicated as C) was significantly associated with a poor disease-free survival rate $(p=0.0299<0.05)$. 
A

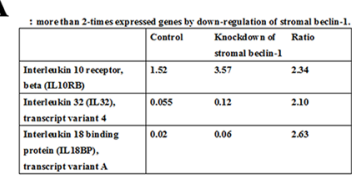

B

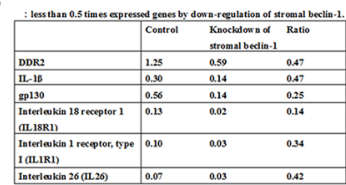

C

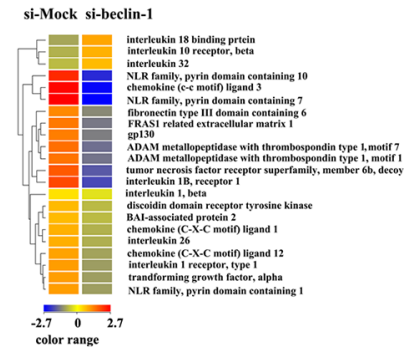

Fig 3. Summary of differentially expressed genes, as indicated by comprehensive microarray analysis, following downregulation of stromal beclin-1 expression, compared with the control MCF7-UE6E7T-2 co-culture system. A: genes with greater than 2-fold expression after downregulation of stromal beclin-1. B: genes with less than 0.5 -fold expression after downregulation of stromal beclin-1. C: hierarchical combined tree of the 22 extracted genes related to inflammation and stromal reconstitution in the cancer microenvironment.

doi:10.1371/journal.pone.0125762.g003

These results indicate that stromal beclin-1 expression may be important for reconstitution of the cancer microenvironment of the stroma thorough the proinflammatory IL- $1 \beta$ pathway and the collagen receptor DDR2.

\section{Discussion}

Immunohistochemical staining revealed that a combination of loss of beclin-1 expression in cancer cells and overexpression of beclin-1 in cancer stromal mesenchymal cells was linked to local cancer recurrence and postoperative lymph node metastasis in invasive ductal carcinoma. There are several conflicting reports regarding the prognostic value of beclin-1 expression in breast cancer. Dong et al. determined that low expression of beclin-1 was associated with a worse 5-year overall survival rate in ER-positive and HER2-negative breast cancer [22]. He

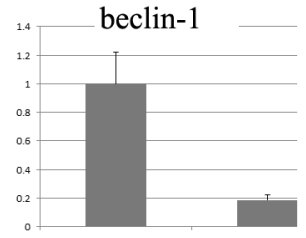
si-Mock si-beclin-1 DDR2

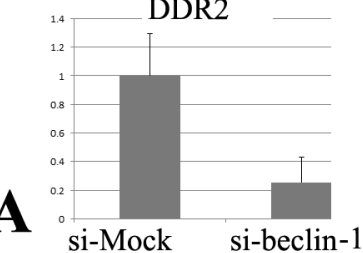

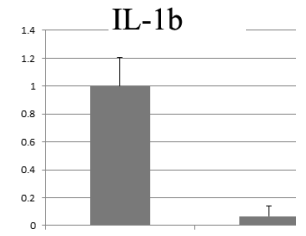

si-Mock si-beclin-1 IL-10RB

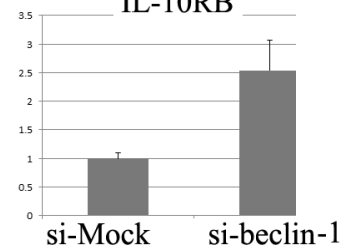

si-Mock si-beclin-1

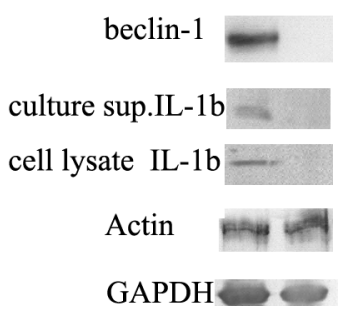

B

Fig 4. Representative quantitative reverse transcription polymerase chain reaction results and immunoblotting. siRNA treatment successfully reduced beclin-1 mRNA (representative data of quantitative RT-PCR) and beclin-1 protein (representative data of Western-immunoblotting) in stromal UE6E7T-2 cells compared to control siRNA treated cells. (A) Downregulation of beclin-1 in UE6E7T-2 stromal cells significantly decreased the expression of the $I L-1 \beta$ and $D D R 2$ genes, whereas that of $I L-10 R B$ was increased in co-cultured MCF-7 and UE6E7T-2 cells. Gene expression changes were computed in triplicate reactions per sample using the $2^{-\Delta \Delta C t}$ method. The data are represented as relative expression values compared with the mRNA expression in siGFP treated cells after the normalization with GAPDH mRNA expression. Error bars indicate standard deviations. All values represent statistically significant fold changes (beclin-1: 0.00014, IL-1ß: 0.00012, DDR2: 0.00011, and IL-10RB: 0.00016). We performed three independent, quantitative RT-PCR experiments using different MCF-7-UE6E7T-2 co-culture systems and obtained similar results. (B) Downregulation of beclin-1 in UE6E7T-2 stromal cells significantly decreased the expression of the IL-1 $\beta$ protein secretion (indicated as culture sup) and production (indicated as cell lysate) in co-cultured MCF-7 and UE6E7T-2 cells. 
et al. reported that high expression of beclin-1 was associated with a favorable prognosis in breast cancer [23]. In contrast, Won et al. found no correlation between beclin-1 expression and the cumulative survival of patients with invasive breast cancer [24]. In the present study, beclin-1 expression in breast cancer cells alone was not significantly associated with patient prognosis, consistent with the data reported by Won et al [24]. We demonstrated that low expression of beclin- 1 in breast cancer cells and high expression of beclin-1 in cancer stromal mesenchymal cells significantly correlates with a poor patient prognosis. Interestingly, lack of beclin-1 expression in cancer cells and overexpression of beclin-1 in stromal cells tends to be associated with local recurrence and postoperative lymph node metastasis.

Microarray analysis indicated that the downregulation of beclin- 1 expression in bone marrow-derived mesenchymal UE6E7T-2 cells, which were co-cultured with MCF-7 cells, resulted in abrogation of the expression of several pro-inflammatory genes and $D D R 2$, while upregulating $I L-10 R B$, which is a receptor for the well-characterized anti-inflammatory cytokine IL-10. As shown in Fig 4, quantitative RT-PCR experiments confirmed that beclin-1 downregulation decreased $I L-1 \beta$ and $D D R 2$ expression, but increased $I L-10 R B$ expression. We speculate that beclin-1 expression in the stromal cells may be important to maintain the levels of IL- 1 and to maintain mesenchymal collagen-binding receptors in the active state in cancer-stromal microenvironments. Importantly, Zhang et al. recently determined that DDR2 post-transcriptionally stabilizes SNAIL1 to promote breast cancer invasion and migration [20]. Moreover, DDR2 expression appears to be significantly associated with breast cancer lymph node metastasis [21]. It is likely that continuous DDR2 expression, which is related to stromal beclin-1 expression, plays a role in local recurrence and lymph node metastasis. In addition, a high IL- $1 \beta$ content within the breast cancer microenvironment is associated with tumor invasiveness [25]. Additional studies are in progress to unravel the molecular pathways by which beclin-1 expression or autophagy contribute to the breast cancer stromal microenvironment. In conclusion, the results from this study indicate that stromal beclin-1 expression could be a potent molecular target in the absence of beclin-1 expression in breast cancer cells.

\section{Supporting Information}

S1 Fig. Representative quantitative reverse transcription polymerase chain reaction results and immunoblotting using co-cultured MDA-MB-157 and UE6E7T-2 cells.

S2 Fig. Representative quantitative reverse transcription polymerase chain reaction results and immunoblotting using co-cultured MCF-7 and UE6E7T-2 cells with si-RNA, 5'CUCAGGAGAGGAGCCAUUUtt-3', to silence the beclin-1 gene. (TIF)

S3 Fig. Representative quantitative reverse transcription polymerase chain reaction results and immunoblotting using co-cultured MCF-7 and UE6E7T-2 cells with si-RNA, 5' GAUUGAAGACACAGGAGGCUU- $3^{\prime}$, to silence the beclin-1 gene. (TIF)

S4 Fig. Immunocytostaining demonstrated that MCF-7 cells exhibited immunoreactivity with AE1/AE3 (anti-multi-Cytokeratin antibody), but not with anti-vimentin antibody. By contrast, UE6E7T-2 cells were stained with anti-vimentin antibody but not with AE1/AE3 antibody (A). At $48 \mathrm{~h}$ after co-culture, the cells were collected by centrifugation, resuspended in $0.1 \mathrm{ml}$ of PBS, dropped on the slide glass, and dried at room temperature. After methanol fixation, the cells were immunostained with antibodies using the ImmPRESS polymerized reporter enzyme staining system (Vector laboratories). We calculated the stained cell numbers in 
representative $\mathrm{x} 200$ power field. We did not observe significant differences in the proportion of AE1/AE3- or vimentin-positive cells between the si-GFP- and si-beclin-1-treated groups. Representative vimentin and AE1/AE3 staining is shown in Figure (B), and the mean and SD are also shown $(\mathrm{C})$. The experiments were performed in triplicate and significance was evaluated using the Student's t-test. Scales bars: $100 \mu \mathrm{m}$

(TIF)

S1 Dataset. The list of downregulated genes in a MCF-7 and mesenchymal stromal cell coculture system by siRNA-mediated silencing of beclin-1 gene expression in mesenchymal stromal cells.

(XLSX)

S2 Dataset. The list of upregulated genes in a MCF-7 and mesenchymal stromal cell co-culture system by siRNA-mediated silencing of beclin-1 gene expression in mesenchymal stromal cells.

(XLSX)

\section{Author Contributions}

Conceived and designed the experiments: TT MF KY. Performed the experiments: AM YK CS TS. Analyzed the data: AM TT. Contributed reagents/materials/analysis tools: AM MF KY.

Wrote the paper: AM TT.

\section{References}

1. Levine B, Kroemer G (2008) Autophagy in the pathogenesis of disease. Cell. 132:27-42. doi: 10.1016/ j.cell.2007.12.018 PMID: 18191218

2. Mizushima N, Levine B, Cuervo AM, Klionsky DJ (2008) Autophagy fights disease through cellular selfdigestion. Nature. 451:1069-1075. doi: 10.1038/nature06639 PMID: 18305538

3. Lorin S, Hamaï A, Mehrpour M, Codogno P (2013) Autophagy regulation and its role in cancer. Semin Cancer Biol. 23:361-379. doi: 10.1016/j.semcancer.2013.06.007 PMID: 23811268

4. Choi AM, Ryter SW, Levine B (2013) Autophagy in human health and disease. N Engl J Med. 368:651-662. doi: 10.1056/NEJMra1205406 PMID: 23406030

5. Liang XH, Jackson S, Seaman M, Brown K, Kempkes B, Hibshoosh H, et al. (1999) Induction of autophagy and inhibition of tumorigenesis by beclin 1. Nature. 402:672-676. PMID: 10604474

6. Kang R, Zeh HJ, Lotze MT, Tang D (2011) The Beclin 1 network regulates autophagy and apoptosis. Cell Death Differ. 18:571-580. doi: 10.1038/cdd.2010.191 PMID: 21311563

7. Li Z, Chen B, Wu Y, Jin F, Xia Y, Liu X (2010) Genetic and epigenetic silencing of the beclin 1 gene in sporadic breast tumors. BMC Cancer. doi: 10.1186/1471-2407-10-98

8. Gong C, Bauvy C, Tonelli G, Yue W, Deloménie C, Nicolas V, et al. (2013) Beclin 1 and autophagy are required for the tumorigenicity of breast cancer stem-like/progenitor cells. Oncogene. 32:2261-72 doi: 10.1038/onc.2012.252 PMID: 22733132

9. Karnoub AE, Dash AB, Vo AP, Sullivan A, Brooks MW, Bell GW, et al. (2007) Mesenchymal stem cells within tumour stroma promote breast cancer metastasis. Nature. 449:557-563. PMID: 17914389

10. Martinez-Outschoorn U, Sotgia F, Lisanti MP (2014) Tumor microenvironment and metabolic synergy in breast cancers: critical importance of mitochondrial fuels and function. Semin Oncol. 41:195-216 doi: 10.1053/j.seminoncol.2014.03.002 PMID: 24787293

11. Maes H, Rubio N, Garg AD, Agostinis $P$ (2013) Autophagy: shaping the tumor microenvironment and therapeutic response. Trends Mol Med. 19:428-446. doi: 10.1016/j.molmed.2013.04.005 PMID: 23714574

12. Takeuchi $T$, Adachi $Y$, Sonobe $H$, Furihata M, Ohtsuki $Y$ (2006) A ubiquitin ligase, skeletrophin, is a negative regulator of melanoma invasion. Oncogene. 25:7059-7069. PMID: 16715130

13. Kurtova AV, Balakrishnan K, Chen R, Ding W, Schnabl S, Quiroga MP, et al. (2009) Diverse marrow stromal cells protect CLL cells from spontaneous and drug-induced apoptosis: development of a reliable and reproducible system to assess stromal cell adhesion-mediated drug resistance. Blood. 114:4441-4450. doi: 10.1182/blood-2009-07-233718 PMID: 19762485 
14. Nishimura K, Semba S, Aoyagi K, Sasaki H, Yokozaki H (2012) Mesenchymal stem cells provide an advantageous tumor microenvironment for the restoration of cancer stem cells. Pathobiology. 79:290306 doi: 10.1159/000337296 PMID: 22688186

15. Soule HD, Vazguez J, Long A, Albert S, Brennan M (1973) A human cell line from a pleural effusion derived from a breast carcinoma. J Natl Cancer Inst. 51:1409-1416. PMID: 4357757

16. Young RK, Cailleau RM, Mackay B, Reeves WJ (1974) Establishment of epithelial cell line MDA-MB157 from metastatic pleural effusion of human breast carcinoma. In Vitro. 9:239-245. PMID: 4471183

17. Takeuchi T, Misaki A, Liang SB, Tachibana A, Hayashi N, Sonobe H, et al. (2000) Expression of T-cadherin (CDH13, H-Cadherin) in human brain and its characteristics as a negative growth regulator of epidermal growth factor in neuroblastoma cells. J Neurochem. 74:1489-1497. PMID: 10737605

18. Towbin H, Staehelin T, Gordon J (1979) Electrophoretic transfer of proteins from polyacrylamide gels to nitrocellulose sheets: procedure and some applications. Proc Natl Acad Sci U S A. 76:4350-4354. PMID: 388439

19. Livak KJ, Schmittgen TD (2001) Analysis of relative gene expression data using real-time quantitative PCR and the $2^{-\triangle \triangle C T}$ method. Methods 25:402-428. PMID: 11846609

20. Zhang K, Corsa CA, Ponik SM, Prior JL, Piwnica-Worms D, Eliceiri KW, et al. (2013) The collagen receptor discoidindomain receptor 2 stabilizes SNAIL1 to facilitate breast cancer metastasis. Nature Cell Biol 15: 677-687. doi: 10.1038/ncb2743 PMID: 23644467

21. Ren T, Zhang W, Liu X, Zhao H, Zhang J, Zhang J, et al. (2014) Discoidin domain receptor 2 (DDR2) promotes breast cancer cell metastasis and the mechanism implicates epithelial-mesenchymal transition programme under hypoxia. J Pathology doi: 10.1002/path.4415

22. Dong M, Wan XB, Yuan ZY, Wei L, Fan XJ, Wang TT, et al. (2013) Low expression of Beclin 1 and elevated expression of HIF-1 $\alpha$ refine distant metastasis risk and predict poor prognosis of ER-positive, HER2-negative breast cancer. Med Oncol. 30:355. doi: 10.1007/s12032-012-0355-0 PMID: 23408367

23. He Y, Zhao X, Subahan NR, Fan L, Gao J, Chen $\mathrm{H}$ (2014) The prognostic value of autophagy-related markers beclin-1 and microtubule-associated protein light chain 3B in cancers: a systematic review and meta-analysis. Tumour Biol. 35:7317-7326. doi: 10.1007/s13277-014-2060-4 PMID: 24838948

24. Won KY, Kim GY, Kim YW, Song JY, Lim SJ (2010) Clinicopathologic correlation of beclin-1 and bcl-2 expression in human breast cancer. Hum Pathol. 41:107-112. doi: 10.1016/j.humpath.2009.07.006 PMID: 19762066

25. Jin L, Yuan RQ, Fuchs A, Yao Y, Joseph A, Schwall R, et al. (1997) Expression of interleukin-1beta in human breast carcinoma. Cancer. 80:421-434. PMID: 9241076 